\title{
Anabases
}

ANABASES Traditions et réceptions de l'Antiquité

4 | 2006

Varia

\section{Jacques Esprit, Sénèque et la clémence}

\section{Guillaume Flamerie de Lachapelle}

\section{OpenEdition}

Journals

Édition électronique

URL : http://journals.openedition.org/anabases/3579

DOI : 10.4000/anabases.3579

ISSN : 2256-9421

\section{Éditeur}

E.R.A.S.M.E.

\section{Édition imprimée}

Date de publication : 1 octobre 2006

Pagination : 111-123

ISSN : 1774-4296

\section{Référence électronique}

Guillaume Flamerie de Lachapelle, « Jacques Esprit, Sénèque et la clémence », Anabases [En ligne], 4 I 2006, mis en ligne le 01 janvier 2012, consulté le 20 octobre 2019. URL : http:// journals.openedition.org/anabases/3579 ; DOI : 10.4000/anabases.3579 
Anabases 4 (2006), p. 111-123

\section{Jacques Esprit, Sénèque et la clémence}

Guillaume Flamerie de LACHAPELLE

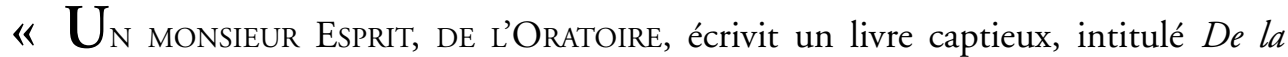
Fausseté des vertus humaines. Cet Esprit dit qu'il n'y a point de vertu ; mais par grâce il termine chaque chapitre en renvoyant à la charité chrétienne... Une telle insolence révolte. Je n'en dirai pas davantage, car je me mettrais en colère ${ }^{1}$. " À lire ce jugement cinglant de Voltaire, suivi de bien d'autres ${ }^{2}$, appréhender Sénèque à travers un écrivain si médiocre paraît superflu. Il nous semble pourtant qu'une telle étude permettra de mettre en évidence l'attrait et la répulsion que suscite tout ensemble le philosophe stoïcien au XVIIe siècle, et cela à partir d'un thème particulier, objet du chapitre I.10 du traité d'Esprit : la clémence.

Précisons brièvement les circonstances dans lesquelles l'ouvrage qui nous occupe voit le jour. Jacques Esprit fréquente, avec La Rochefoucauld, le cercle de Mme de Sablé avant de se retirer dans son Béziers natal pour élaborer le traité qui couronne une exis-

1 Voltaire, Dictionnaire philosophique, art. "Fausseté des vertus humaines".

2 H. Busson, La Religion des Classiques (1660-1685), Paris, 1948, p. 211 : "Les dissertations pâteuses, monotones et compassées de Jacques Esprit lassent la patience ; J. LAFOND, La Rochefoucauld. Augustinisme et littérature, Paris, 1977, p. 109 ; p. 126 : "didactisme simplifiant " ; même A. SouCAILlé, "Notice biographique et littéraire sur l'académicien Esprit ", Bulletin de la société archéologique, scientifique et littéraire de Béziers 4(2) (1867), p. 45-89, qui admire d'ordinaire Esprit, blâme un traité si révoltant (p. 60-69). La Fausseté a pourtant des défenseurs, comme A. BALUfFE, "Un oublié du XVII siècle. J. Esprit (1611-1678) ”, Revue bleue 2 (1894), p. 133-140 ou L. BATTEREL, Mémoires domestiques pour servir à l'histoire de l'Oratoire, Paris, 1903, p. 267-278 (surtout p. 276-277). 
tence vouée à l'érudition et à l'étude des mœurs : La Fausseté des vertus humaines, paru en 1678 , un an après sa mort ${ }^{3}$. Cette monographie est construite de façon répétitive, les vertus se trouvant minutieusement examinées les unes après les autres. Esprit rejette chacune d'elles au nom des motifs déshonorants qui sont ordinairement à l'origine de sa pratique, avant de la réhabiliter finalement en soulignant qu'elle peut atteindre à la perfection, à condition d'être exercée chrétiennement. Questionner les vertus héritées des Anciens est une pratique courante à la fin du XVII e siècle, surtout dans les milieux jansénistes dont Esprit fait partie ${ }^{4}$, mais il en est incontestablement le théoricien le plus systématique, et y montre une certaine finesse.

Pour nourrir sa réflexion sur la clémence, Esprit ne s'appuie certes pas exclusivement sur les Anciens : telle phrase, par exemple, semble reprise de Montaigne ${ }^{5}$. Par ailleurs, le Grand Siècle n'est pas seulement le siècle de Cinna, et les remises en cause de la clémence ont déjà fleuri sous la plume de Richelieu, qui la considère comme contraire à la justice ${ }^{6}$, ou de La Rochefoucauld, selon lequel elle couvre d'un voile honnête des motivations contestables ${ }^{7}$. Le cadre de pensée dépasse donc l'Antiquité. Il n'en reste pas moins que presque tous les exemples sont empruntés à cette période ${ }^{8}$, et

3 Nous citons le traité d'Esprit d'après la réédition de 1996, précédée d'une préface de $\mathrm{P}$. QUIGNARD, en indiquant le livre, le chapitre, et enfin la page. Pour les textes anciens, nous suivons la Collection des Universités de France; nous empruntons les traductions du De Clementia à Fr.-R. Chaumartin (éd.), Sénèque. De la Clémence, Paris, 2005.

Les vertus païennes sont l'objet d'un débat déjà vieux de plusieurs décennies quand Esprit publie son traité : procèdent-elles d'un sentiment honorable, malgré l'ignorance de la Révélation, comme le pense La MOTHE LE VAYER, De la Vertu des payens (1641), ou bien relèvent-elles de mobiles blâmables, comme le veut JANSÉNIUS ? Dans sa préface, Esprit précise cependant que ce débat ne le concerne pas (p. 78). Montaigne, Essais, III,8 (“ De l'Art de conférer ”) : "L'horreur de la cruauté me rejette plus en avant en la clémence qu'aucun patron de la clémence ne saurait m'attirer ", à rapprocher d'Esprit, I,10, p. 175: «L'horreur qu'on a de la cruauté contribue encore beaucoup à nous faire aimer la clémence ". Montaigne est l'un des auteurs modernes les plus cités du traité, et Esprit s'en prévaut dès sa préface (p. 79). Fr. BillaCOIS, "Clémence ou justice, un débat sur la fonction royale aux débuts de l'absolutisme ", Revue historique de droit français et étranger 44 (1966), p. 444-450 ; Fr. Hildesheimer, "Pardonner ou châtier ? Richelieu ou l'impossible clémence ", in Le Pardon, ed. J. Hoareau-Dodinau, X. Rousseaux \& P. Texier, Limoges, 1999, p. 425463.

7 La Rochefoucauld, Maximes, 15 et 16 (éd. J. TRUCHET) ; autres exemples dans M. Bouvier, La Morale classique, Paris, 1999, p. 530-531.

8 Les seuls exemples empruntés à l'époque d'Esprit concernent les courtisans (I,10, p. 178) et le duel (I,10, p. 183), qui servit si souvent aux philosophes du XVII à illustrer leurs principes : cf. e. g. DESCARTES, Lettre à la Princesse Élisabeth de janvier 1646, sur le librearbitre. Esprit consacre d'ailleurs tout un chapitre (II,11) à la bravoure dans les duels. 
que tous les auteurs profanes explicitement cités sont grecs ou romains 9 . De fait, c'est visiblement à un dialogue qu'Esprit se livre : ce sont les grands héros cléments - Alexandre, César, Auguste - et le Sénèque du De Clementia qui se trouvent visés, même si ce dernier n'est mentionné qu'à une seule reprise dans le chapitre consacré à la clémence. Aussi bien a-t-on parlé d'un " anti-sénéquisme total " d'Esprit ${ }^{10}$, qui ne détonnerait guère à une époque où l'adhésion au néostoïcisme recule, où l'étoile de Sénèque pâlit ${ }^{11}$. Le frontispice de l'édition de 1678 est à cet égard éloquent : Sénèque, grimaçant derrière un masque avenant, un livre et un poignard à la main, est repoussé par un jeune homme qui rejoint une allégorie de Veritas, avec ce commentaire : Non faciet iam larua pares, dont le sens est obscur. Sans doute faut-il comprendre : "Ce fantôme (ou " ce masque ") ne fera plus de semblables 12 " ou bien "Ce fantôme ne rendra plus égales les fausses vertus et les vraies ${ }^{13}$. " La balance présente sur la cuirasse de Veritas suggère en tout cas que Sénèque doit désormais cesser d'être l'arbitre des vertus. Quel que soit le sens exact de la maxime inscrite sous le frontispice, maxime qu'Esprit, surpris par la mort, n'eut peut-être pas le loisir de choisir, le livre se trouve placé sous le signe du combat contre le mensonge.

9 Sont mentionnés explicitement : Platon (4 fois) ; Plutarque (3) ; Aristote (2) ; Cicéron (2) ; Sénèque (1) ; Tite-Live (1) ; Quinte-Curce (1) ; Diogène Laërce (1). Comme il est d'usage, les citations ne sont pas en langue originale (dans l'ensemble de l'œuvre, Esprit ne cite que rarement le latin, et seulement pour des vers : I,1, p. 85 ; I,14, p. 221 ; II,13, p. 418...). Esprit résume les conceptions de certains auteurs, ou les cite avec un degré de fidélité variable : les Écritures sont reproduites littéralement, alors que les Anciens peuvent être cités en substance (I,10, p. 186, d'après Cicéron, De Officiis, I,88). La pensée de TiteLive, II,3,3-4, est enfin citée de façon détournée (I,10, p. 184) : Esprit attribue à l'historien une conception exprimée par des aristocrates désireux de renverser la jeune République, avec lesquels le Padouan prend nettement ses distances.

10 P. QUiGNARD [n. 3], p. 40-41.

11 M. Spanneut, "Permanence de Sénèque le philosophe ", Bulletin de l'Association Guillaume Budé(1980), p. 399.

12 On peut arriver à cette traduction en considérant pares comme un substantif complément d'objet, ou comme un adjectif attribut d'un complément à l'accusatif sous-entendu (" Il ne rendra plus les gens pareils à lui »). Les traductions que propose P. QUIGNARD [n. 3], p. 38, sont fantaisistes : "Désormais le masque ne fera pas le poids " ou "Les fantômes ne remboursent plus leur dette. "

13 Dans ce cas, peut-être Esprit a-t-il voulu détourner ironiquement le dogme stoïcien de l'égalité des vertus : Sénèque, De Clementia, I,5,3 ; Lettres à Lucilius, 66,10 ; 113,16 ; Stoicorum Veterum Fragmenta, III,295-304. 


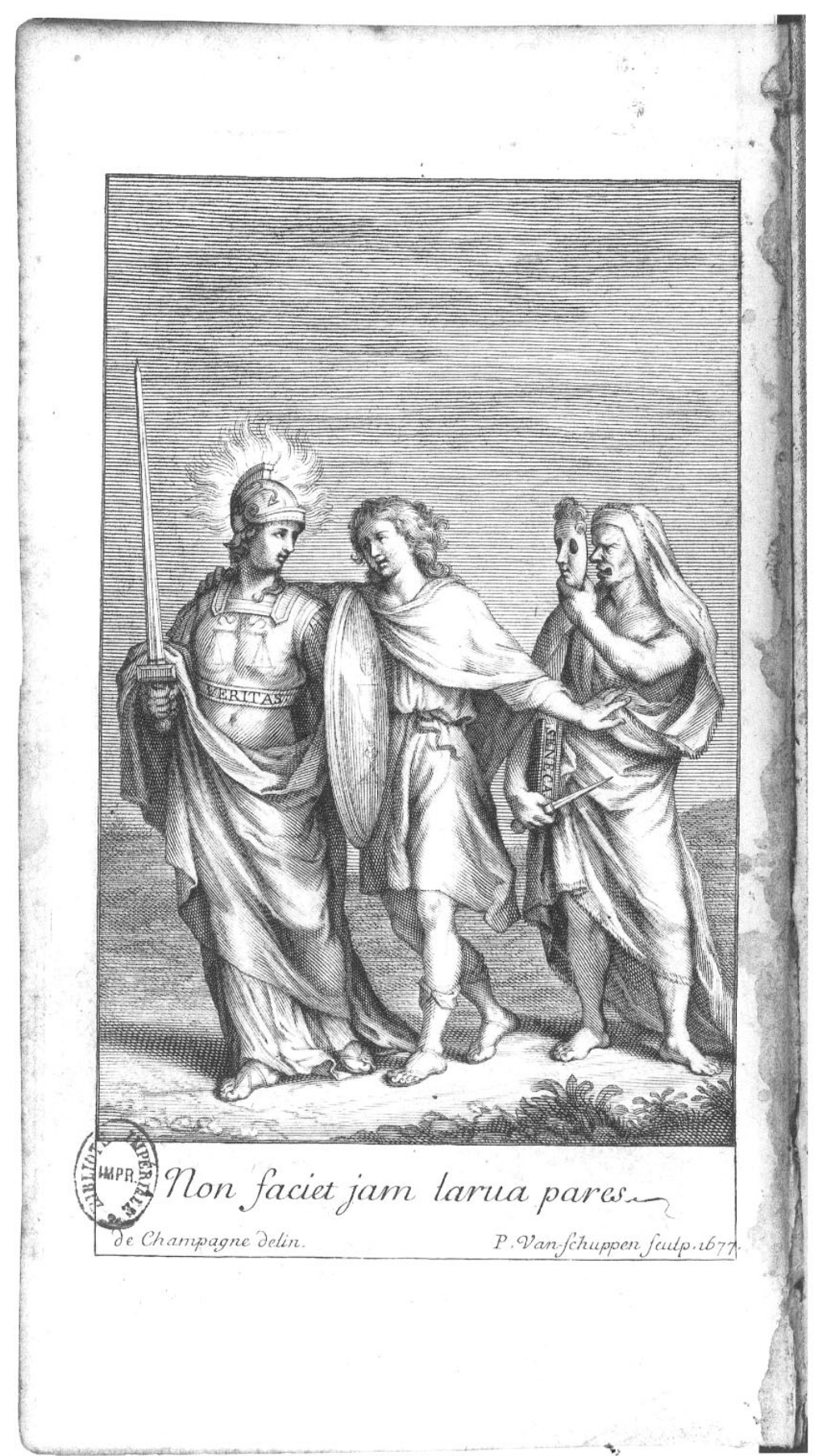

BnF - RES - RES-R-2052, frontispice / BnF-DRE-Utilisation réglementée 
L'objet du présent article est de montrer comment Esprit, s’il s'attache à rejeter les positions de Sénèque, en vient à réemployer les arguments de ce dernier ${ }^{14}$ : loin d'être un pur jeu sophistique, cette pratique témoigne peut-être d'une tension entre sa fréquentation assidue des Anciens 15 et sa conception de la grâce, inconciliable avec leurs vues.

I. Esprit adopte différentes attitudes face aux arguments païens et sénéquiens. Il estime que certains sont justes, mais que le Stoïcien n'en tire pas toutes les conséquences, ce qui invalide la thèse finale de ce dernier et prouve son incohérence ; que d'autres travestissent une sombre réalité sous des dehors flatteurs, et doivent être dénoncés ; que d'autres enfin sont tout à fait contraires à la vérité. Ces réfutations n'excluent pas les blâmes dirigés contre la personne même de Sénèque.

I.1. Esprit combat d'abord le Stoïcien avec ses propres armes. Ainsi, la seule citation explicite du De Clementia sert à affaiblir la position du Cordouan :

Auguste, dit Sénèque, après tant de proscriptions et de meurtres, donna la vie à Cinna, fit cette action de clémence afin qu'on le crût clément ; pour moi [i. e. Sénèque] je ne saurais appeler clément un homme qui se lasse d'être cruel (I,10, p. 178).

Esprit cite ici en substance deux passages du De Clementia (I,9,2 et I,11,2), mais passe délibérément sous silence les phrases élogieuses consacrées au règne d'Auguste $(\mathrm{I}, 10)$ entre le récit du pardon de Cinna $(\mathrm{I}, 9)$ et la distinction entre clementia et lassa crudelitas $(\mathrm{I}, 11,2)$; Esprit fait aussi de la clémence d'Auguste une pure manœuvre politique, sans signaler que le prince était alors incapable de tuer Cinna (I,9,3 : non poterat), qu'il souffrait sincèrement en infligeant des punitions $(\mathrm{I}, 10,3)$ et que sa méconduite précédente lui causait un profond remords $(\mathrm{I}, 11,1)$. En juxtaposant brutalement grandeur apparente et sordide calcul, en estimant que la clémence ne sert qu’à soutenir une "tyrannie ", Esprit laisse croire que Sénèque, conscient que la clémence d'Auguste est hypocrite tout en persistant à louer cette vertu, se montre inconséquent 16 .

14 Ce type d'enquête a été mené pour d'autres auteurs ; citons en particulier JULIEN-EYMARD D’ANGERS, "Réfutation et utilisation augustiniennes de Sénèque et du Stoïcisme dans L'homme criminel (1644) et L'homme chrétien (1648) de l'oratorien J.-F. Senault ", in Recherches sur le Stö̈cisme aux XVIe et XVII siècles, Hildesheim, 1976, p. 373-405.

La culture et l'intelligence d'Esprit ont parfois été mises en doute : le jésuite R. RaPIN, dont la charité est parfois limitée, porte dans ses Mémoires, éd. L. AubinEAU, Paris, 1865, un jugement particulièrement peu flatteur (t. I, p. 91 ; p. 101 : "parleur agréable, d'une capacité superficielle»; p. 104 ; t. III, p. 235), mais l'érudition dont témoigne la Fausseté, ainsi que divers témoignages contemporains (Voiture ou Balzac, cf. A. BALUfFe, "Un oublié... " [n. 2], p. 137-138) interdisent du moins de mettre en cause sa culture.

Esprit, II,21, p. 474, lui reproche explicitement de ne pas tirer la leçon de ses propres connaissances : conscient de la méchanceté naturelle de l'homme, le philosophe n'en croit pas moins orgueilleusement à la puissance de sa raison ; en II,14, p. 424-427, Sénèque est réfuté par lui-même, au sujet de la grandeur d'âme. 
En fait, Sénèque avait mentionné la clémence imparfaite d'Auguste pour l'opposer à celle, authentique et véritable, du jeune Néron qui, au moment du De Clementia, laisse encore espérer une ère de mansuétude après la fin sanglante du règne de Claude. Esprit a bien sûr beau jeu d'abattre cet argument, en évoquant la cruauté dont fera preuve l'élève du Cordouan par la suite. Quel meilleur exemple de la vanité de Sénèque que son propre échec ${ }^{17}$ ? Esprit emprunte au De Clementia la substance de discours rassurants et emplis de satisfaction prononcés par Néron ${ }^{18}$, et met ces propos sur le compte de la dissimulation. En niant que le fils d'Agrippine ait été sincèrement clément avant d'être en proie à son naturel malade ${ }^{19}$, Esprit sous-entend que Sénèque a été complice de l'entreprise de son disciple, ou qu'il en a été la dupe. Dans un cas comme dans l'autre, c'est affaiblir la position du précepteur.

I.2. Après avoir placé Sénèque devant ses échecs et ses contradictions, Esprit entreprend de le démasquer, en montrant comment la clémence qu'il prône repose sur des conceptions insoutenables. La critique la plus massive qu’adresse Esprit à la clémence est courante en son siècle ${ }^{20}$ : «C'est une ignorance de l'utilité et de la nécessité de la justice ", cette dernière étant fondamentale selon saint Augustin ${ }^{21}$. Esprit substitue cette opposition entre clémence et justice à celle qu'établissait le Stoïcien entre clémence et cruauté. Pour Esprit, cette dernière antinomie est fallacieuse 22 , et dissimule par son caractère spectaculaire le seul contraste qui importe : celui qui existe entre clémence et justice ${ }^{23}$. Pour

17 Esprit a la partie facile en évoquant Néron, qui est pour ses lecteurs l'incarnation de la cruauté. Cette dénonciation implicite de l'impuissance de Sénèque à contrôler son élève est exprimée sans ambages par La Rochefoucauld dans une de ses Maximes supprimées (21).

Esprit, I,10, p. 181 : "Il se glorifiait justement qu’il n'avait pas été répandu une seule goutte de sang dans tout l'Empire ", paraphrase de De Clementia, I,11,3 : gloriatus es nullam te toto orbe stillam cruoris humani misisse.

19 Telle est pourtant la version généralement retenue par les Anciens : cf. Suétone, Néron, 26,1; Aurélius Victor, Livre des Césars, 5,2-4.

20 Cf. [n. 6]. Même Senault, Le Monarque ou les devoirs du souverain, Paris, 1661, qui consacre à la clémence du prince un chapitre entier (lequel relève d'une paraphrase assumée du De Clementia), conclut sur cet avertissement révélateur : "La Prudence doit accorder la Clémence avec la Justice... ; elle doit terminer le différend entre les deux Sœurs. " $\mathrm{La}$ justice, "sans laquelle, dit Augustin, les républiques et les empires sont de grandes sociétés de brigands " (I,10, p. 182), citation tirée de la Cité de Dieu, IV,4(150). Opinion courante au XVII siècle : cf. A. GODEAU, "Lidée du bon magistrat en la vie et en la mort de M. de Cordes ", in Euvres chrétiennes et morales en prose, Paris, 1658, t. I, p. 471 : " Il savait qu'il y avait une clémence criminelle, et que Dieu, dont il avait l'honneur d'exercer l'office, est aussi saint quand il fait pleuvoir le souffre et le feu sur Sodome et quand il nourrit les Enfants d'Israël dans le désert "; A. N. Amelot de La Houssaie, La Morale de Tacite, Paris, 1686, p. 245.

23 I,10, p. 175-176. Sénèque place en opposition la clémence au début du livre I du De Clementia, et la cruauté à la conclusion de ce même livre ; en II,4,1, il définit la cruauté comme l'exact contraire de la clémence. La clémence et la justice, étant deux vertus, ne peuvent entrer en contradiction dans l'esprit du Stoïcien. 
mettre Sénèque en difficulté, Esprit identifie justice et respect des lois, sans retenir la conception sénéquienne, qui fait de la clémence l'acte le plus juste possible, quitte à contredire à l'occasion la loi formelle ${ }^{24}$. Non content de dénoncer le caractère superficiel de l'opposition entre clémence et cruauté, qui dissimule la profonde incompatibilité de la clémence et de la justice, Esprit lance des attaques contre l'argument de la dignité royale, qui doit contraindre le souverain à se montrer clément :

Il y a des occasions où la clémence des rois n'est qu'une vaine ostentation de leur puissance souveraine ; car comme rien ne flatte tant l'orgueil de l'homme que l'élévation, rien aussi ne plaît tant à sa vanité que ce qui la lui rend présente et qui la fait voir aux autres. Or la clémence montre que les souverains sont au-dessus des lois, et qu'ils ont le pouvoir non seulement d'ôter, mais aussi de donner la vie (I,10, p. 181-182) 25.

La gloire et la conscience de son rang (dignitas), tenues par Sénèque pour de nobles sentiments qui obligent le prince ${ }^{26}$, deviennent pour le janséniste Esprit une vanité blâmable, qui ne saurait en rien justifier une attitude clémente ${ }^{27}$. Saper ces arguments est d'autant plus facile que, dans une large mesure, Sénèque les présentait sous une forme hyperbolique pour mieux persuader son jeune élève ${ }^{28}$. La clémence, issue du pur orgueil du prince, bafoue dès lors l'intérêt public $\left(I, 10\right.$, p. 182) ${ }^{29}$, alors même que c'était la première raison pour laquelle Sénèque l'appelait de ses vœux ${ }^{30}$. De fait, d'après Esprit, l'auteur des Lettres à Lucilius lui-même n'est pas à l'abri de l'orgueil et

24 De Clementia, II,7,3 : la clémence " n'accomplit aucune de ces actions avec le sentiment qu'elle agit en deçà du juste, mais avec le sentiment que sa décision est la plus juste » (Nibil ex his facit tamquam iusto minus fecerit, sed tamquam id quod constituit iustissimum sit). Les derniers mots rappellent De Clementia, I,5,4 : Occidere contra legem nemo non potest, seruare nemo praeter me. Les attaques contre la générosité du vainqueur clément, uniquement soucieux de laisser son nom dans l'histoire, sont du même ordre (I,18, p. 244-246). De Clementia, I,3,3 ; I,5,4 ; I,17,3 ; I,19,1 ; I,20,3. Il est même nécessaire de rendre sa clémence ostentatoire et éclatante, afin qu'elle conserve son utilité : I,21,4. Cette idée se trouve aussi dans le Pro Marcello, 9 et 20 de Cicéron, auquel Esprit fait allusion (I,10, p. 184).

Sur les attaques jansénistes contre la gloire et l'ensemble des vertus aristocratiques, cf. P. BÉNICHOU, Morales du grand siècle, Paris, $1988^{2}$, p. 128-148.

Sénèque n'omet pas de signaler les dangers d'une vaine gloire : De Clementia, I,1,3 ; $\mathrm{I}, 3,5 \ldots$

29 Tels sont déjà les griefs adressés communément contre la clémence au début du siècle : cf. Fr. Billacois, “Clémence ou justice..." [n. 6], p. 447-448. LeibNiZ, examinant, dans une lettre de septembre 1708 à l'électrice de Brunswick-Lunenbourg, le traité de Des Bans abrégeant l'œuvre d'Esprit, rejettera cet argument : L.-A. FOUCHER de CAREIL (éd.), Lettres et opuscules inédits, Paris, 1854 (rep. Hildesheim, 1975), p. 164-165.

De Clementia, I,3,2. 
de la fatuité 31 . Au mieux la clémence, ainsi conçue comme un devoir inhérent à la charge royale, n'est qu'un acte mécanique qui se trouve, par cette raison même, inférieure à l'humble qualité de douceur 32 .

Le consensus et l'amour mutuel entre l'empereur et ses sujets que vante Sénèque sont seulement, selon Esprit, les marques de flatteries réciproques entre le prince soucieux de conserver son pouvoir et des criminels heureux de leur impunité :

D’un côté un prince... se persuade aisément que sa douceur est une véritable et vertueuse clémence, et il est confirmé dans son opinion par les louanges empoisonnées de ses flatteurs; et de l'autre, tous ceux à qui le prince a sauvé la vie, tous leurs proches et tous leurs amis ne manquent jamais à prendre pour une grande vertu la bonté qui l'a disposé à la leur accorder, et ils ne se soucient guère si l'impunité des crimes est contraire à la justice, et si elle blesse le bien public (I,10, p. 175).

Esprit, mêlant volontiers attaques ad hominem et considérations plus générales 33 , compte peut-être Sénèque, " qui ne pouvait supporter les mœurs des autres hommes, $<$ mais qui> trouve celles de Néron dignes d'admiration 34 " parmi les flatteurs qui entretiennent le prince dans ses illusions ; le prologue du De Clementia, où Sénèque déclare qu'il jouera le rôle d'un simple miroir pour satisfaire la uoluptas du jeune prince, pouvait porter Esprit à formuler un tel jugement, même si le Cordouan exclut toute flagornerie de la part des concitoyens de Néron et de lui-même ${ }^{35}$. La clémence, si salubre chez Sénèque, provoque en revanche la ruine, du point de vue d'Esprit 36.

I.3. Esprit reproche non seulement à Sénèque de déformer la réalité, mais aussi de la nier. C'est ce que montre l'examen des causes exogènes de la clémence du souverain. Pour le Stoïcien, elle est ce à quoi tout homme a droit par son simple titre d'homme ${ }^{37}$; pour Esprit, elle est réservée aux Grands, dont elle encourage les brigues et les vices ${ }^{38}$.

31 Esprit, I,25, p. 288-289, reproche ainsi à Sénèque, Lettres à Lucilius, 7,12, de penser que le Sage méprise l'approbation de la foule au profit de son propre jugement : "Celui qui fait des actions de probité à l'insu du monde, les fait par un désir véritable d'être approuvé, puisque sa plus forte passion étant d'être approuvé de lui-même, il recherche l'approbation qui lui est la plus précieuse, et qu'il préfere à tous les applaudissements publics. » Esprit, I,11, p. 189-190.

Ailleurs, c'est le goût du luxe de Sénèque, en contradiction avec ses écrits, qui sera dénoncé : II,20, p. 462.

Esprit, I,2, p. 111-112.

Des concitoyens de Néron : I,1,7 ; I,13,5; I,19,7 ; de Sénèque lui-même : II,2,2.

Esprit, I,10, p. 174, imité du De Clementia, I,4.

De Clementia, I,1,3.

$\mathrm{I}, 10$, p. 183. Le moraliste s'indigne régulièrement de ce que, dans une monarchie, les Grands échappent trop facilement aux rigueurs des lois (I,4, p. 129...). 
La clémence marque-t-elle pour Sénèque la toute-puissance du roi 39 ? Selon Esprit, elle fait au contraire du souverain la proie souvent impuissante des flatteurs et des sophistes venus solliciter une grâce ${ }^{40}$. La monarchie est pour Sénèque une noble servitude ${ }^{41}$, alors que la clémence n'est souvent attribuée, selon Esprit, qu'en raison de la lassitude d'un prince avide de plaisirs et anxieux de se défaire de sa charge quand il a affaire à un solliciteur trop pressant ${ }^{42}$. En insistant sur la valeur exemplaire de la peine, en soulignant, à la suite de Platon et de saint Augustin ${ }^{43}$, que «l'intérêt public rend la cruauté des lois légitime et fait que tout le monde approuve qu’on roue les scélérats ", Esprit s'éloigne encore de Sénèque, qui jugeait dans le De Clementia que le spectacle de supplices risquait avant tout d'inciter au crime ${ }^{44}$.

II. Tout cela ne signifie pas qu'Esprit ait fait table rase de la pensée de Sénèque. Distinguer les vertus véritables des vices qui en ont l'apparence fut une attitude stoïcienne, avant d'être janséniste ${ }^{45}$ : de cette analogie de méthode résulte parfois une communauté de conceptions.

Les exemples, tout d'abord, sont souvent similaires. Le Stoïcien adressait luimême à Alexandre, César ou Auguste les critiques que formule Esprit : le premier est un personnage bouillonnant capable d'actes fort cruels 46 ; la clémence du deuxième est une feinte pour asseoir son pouvoir ${ }^{47}$; le troisième enfin n'a été clément que tardivement ${ }^{48}$.

39 De Clementia, I,1,2-3 ; I,5,6 et passim. Cf. A. BorGO, "Questioni ideologiche e lessico politico nel De Clementia di Seneca ”, Vichiana 14 (1985), p. 280-282.

40 Esprit, I,10, p. 183.

41 De Clementia, I,8,1 : nobilis seruitus; cf. Tr. ADAM, Clementia principis. Der Einfluß hellenistischer Fürstenspiegel auf den Versuch einer rechtlichen Fundierung des Prinzipats durch Seneca, Stuttgart, 1970, p. 27-31. Esprit, II,19, p. 454, critiquera précisément la présentation fallacieuse de la monarchie comme un "esclavage honorable". Platon, Protagoras, 324 b ; Lois, IX 854 e ; 862 e ; XI 934 b ; Saint Augustin, Cité de Dieu, XIX,16(383).

De Clementia, I,23,1 ; cf. M. Ducos, “La réflexion sur le droit pénal dans l'œuvre de Sénèque ", Helmantica 44 (1993), p. 451-455. En cela, sa position a évolué depuis le De Ira, I,19,7, que cite Esprit (cf. M.T. GRIFFIN, Seneca, a Philosopher in Politics, Oxford, $1992^{2}$, p. 169).

46 Esprit, II,14, p. 426-429, se déclarera lui-même d'accord sur ce point avec Sénèque, De Beneficiis, I,13, et Lettres à Lucilius, 94,62-63 ; la critique se trouvait déjà dans le De Clementia, I,25,1.

47 Sénèque, De Beneficiis, V,16,5.

48 De Clementia, I,11,1-2 : cf. supra, et P. JAL, “Images d'Auguste chez Sénèque ", Revue des Études Latines 35 (1957), p. 242-264 (surtout à partir de la p. 256). 
Mais Esprit et Sénèque se rejoignent parfois aussi sur le fond. Tous deux rejettent une clémence dévoyée qui ne consisterait qu’à accorder l'impunité à tous les crimes 49 . La définition même que donne Esprit de la clémence souhaitable est proche de celle de Sénèque ${ }^{50}$. Les caractéristiques de la clémence vertueuse semblent en fait largement empruntées au Stoïcien : elle est pour Sénèque comme pour Esprit un moyen de réserver un traitement équitable à l'auteur d'une faute involontaire ${ }^{51}$; la peine infligée n'a pas vocation à compenser le dommage subi par la victime ${ }^{52}$. La clémence est enfin le signe de l'affection du souverain pour son peuple, elle montre que le lien qui unit l'un à l'autre est de la même nature que l'amour d'un père pour ses enfants. Esprit cite à l'appui de sa position les Écritures (I,10, p. 186) ${ }^{53}$, mais il est clair que ce thème est largement sénéquien ${ }^{54}$. Dans une lettre à Madame de Sablé, Esprit révélait d'ailleurs son souhait de montrer dans son ouvrage un aspect de la beauté des morales anciennes 55 .

III. La déformation malicieuse des arguments de Sénèque, leur réutilisation dissimulée, les attaques personnelles auxquelles se livre Esprit pourraient laisser croire à une entreprise de démolition assez vaine et gratuite. En réalité, les divergences entre Sénèque et Esprit ont un caractère profond. La critique de la clémence de Sénèque procède notamment de l'anthropologie pessimiste d'Esprit, qui concerne d'abord l'absence de toute sincérité : Sénèque écrit dans le De Clementia que «nul ne peut porter un masque longtemps $56_{\text {"; }}$ pour Esprit au contraire, il est pratiquement impossible à un homme d'ôter son masque et de découvrir sa nature au grand jour ${ }^{57}$. Plus profondément, la notion

49

De Clementia, I,2,2 ; Esprit, I,10, p. 182.

Esprit, I,10, p. 184 : «La propre fonction de la clémence <est> de remettre entièrement les peines qu'on a méritées, ou d'en adoucir la rigueur ", à rapprocher du De Clementia, II,3,2 : ... moderationem aliquid ex merita ac debita poena remittentem, "Une forme de modération faisant quelque remise d'une peine méritée et due ».

De Clementia, I,2,1 ; II,6,3. Cette idée était commune à de nombreux penseurs antiques : Thucydide, III,40,1 ; Platon, Lois, IX 865 a-866 d...

Sénèque, De Ira, I,19,7 ; l'idée de compensation est également absente du De Clementia. Proverbes, XX,28 : clementia thronus regius roboratur.

De Clementia, I,14, et passim. Cf. G. PIERI, "Pouvoir paternel et politique ou patria potestas et politique (à propos du De Clementia de Sénèque) ", Mémoires de la Société pour l'Histoire du Droit et des Institutions des anciens pays Bourguignons, comtois et romands 58 (2001), p. 255-263.

5 Lettre du 29 novembre 1673 : «Faire voir ce qu'il y a de plus beau et de plus exquis dans la philosophie morale des Platoniciens, Péripaticiens (sic), Cirénaïques, Ciniques, Stoïciens et Épicuriens. »

De Clementia, I,1,6.

Cf. e. g. le chap. I,3, consacré à la sincérité. On sait aussi l'attention que La Rochefoucauld a portée à ce thème, sans faire preuve du même rigorisme qu'Esprit (cf. J. LAFOND, La Rochefoucauld [n. 2], p. 59-63 puis p. 100-112). 
de progrès moral est mise en doute : un homme dépourvu de la grâce, que sa volonté est impuissante à obtenir à elle seule, ne saurait se corriger car ses inclinations naturelles sont trop fortes 58 .

Par ailleurs, la clémence d'un homme naturellement indulgent ne contient aucun mérite particulier ${ }^{59}$. Mais même un individu à la bonté innée se trouve incapable de résister à son ambition, qui le contraindra à faire preuve de férocité, non par tempérament, mais par calcul : dans les deux cas, la cruauté est également inéluctable. Aussi le modèle défini par Sénèque, qui souligne à de nombreuses reprises que la clementia de Néron lui est naturelle et, pour cela, durable ${ }^{60}$, ne saurait-il être conservé. Il n'est pas impossible qu'Esprit fonde son point de vue, au moins pour partie, sur Platon, cité abondamment et avec faveur tout au long du traité 61 : dans le Politique, le fondateur de l'Académie esquissait un gouvernement idéal, aux mains d'un seul homme, plus capable que les lois impersonnelles d'adapter la peine aux cas individuels dans le sens de la clémence, avant de se résoudre à l'évidence : un homme si parfait n'existe pas, de sorte que les lois doivent demeurer la norme ${ }^{62}$, ce qui est aussi la conclusion d'Esprit.

C'est pourquoi Esprit, d'un point de vue intellectuel, se place en définitive du côté d'un Stoïcisme plus intransigeant que celui de Sénèque, tel celui qu'incarne Chrysippe :

Un prince sage, disent les Stoïciens, ne doit point avoir cette pitié efféminée qui ne peut souffrir qu'on punisse les crimes, et il doit pour l'ordinaire préférer les rigueurs salutaires des lois à la douceur que lui inspire la bonté de son naturel (I,10, p. 186).

Esprit indique comme référence Diogène Laërce, VII 63, mais la première partie de ce passage fait aussi penser à la distinction opérée par Sénèque entre misericordia et clementia 64, qu'Esprit connaît et cite par ailleurs (I,15, p. 227-228) : c'est d'ailleurs Sénèque (II,5,1), et non Diogène Laërce, qui assimile pitié et effémination ; c'est aussi Sénèque, non Diogène Laërce, qui s'intéresse spécifiquement à la question du prince. Pourtant, l'auteur du De Clementia n'est pas cité : en prônant la clémence comme une

58 Sur cet aspect de l'œuvre d'ESPRIT, cf. e. g. R. KERVILER, Le chancelier Séguier, Paris, 1875², p. 558-559.

59 Esprit considère souvent qu'une vertu, pour mériter ce nom, doit s'élever au-dessus de l'inclination naturelle : I,9, p. 170 ; I,11, p. 189-190..., ce qui est au demeurant une idée tout à fait stoïcienne : cf. Lettres à Lucilius, 90,46.

De Clementia, I,1,6 ; I,1,7 ; II,1,1 ; II,2,1.

61 Préface, p. $75 ; \mathrm{I}, 12$, p. 209 ; I,22, p. $270 ; \mathrm{II}, 3$, p. $333 \ldots$

62 Platon, Politique, 294 a-c; cf. Fr. D'AGostino, Epieikeia, il tema dell'equità nell'antichità greca, Milan, 1973, p. 54-57.

63 Il s'agit du $₫ 123$; cf. aussi Aulu-Gelle, Nuits attiques, XIV,4,2-4 ; De Clementia, I,2,1 ; II,5,2. La pensée vétéro-stoïcienne est sans doute plus nuancée que ne le dit Diogène Laërce, mais ce qui nous importe est la perception qu'en a Esprit. 
règle de gouvernement, au détriment des lois, il s’est écarté des limites salutaires imposées par sa propre École.

Comme toujours, Esprit conclut sa démonstration en soulignant l'infinie supériorité des vertus inspirées par l'amour du Christ. On a reproché à ces passages d'être froids et hypocrites, uniquement destinés à atténuer la dimension scandaleuse du propos ${ }^{65}$. Sans nous prononcer sur ce point, remarquons que la frontière établie par Esprit entre la clémence stoïcienne et la clémence chrétienne rend largement compte des critiques qu'il a formulées auparavant :

Il ne faut pas que les rois qui veulent être vertueusement cléments se renferment dans les considérations qui font agir ordinairement les princes sages et raisonnables; il faut qu'ils s'élèvent plus haut, et que dans toutes les actions de clémence qu'ils font, ils se proposent d'imiter Dieu qui a fait non seulement paraître, mais éclater la sienne dans le rachat des hommes qu'il a délivrés des supplices éternels qui leur étaient préparés (I,10, p. 186-187).

Après avoir blâmé une fois encore la dimension machiavélienne et calculatrice de la clémence païenne ${ }^{66}$, Esprit critique au fond surtout l'orgueil de la raison. Pour Sénèque, la clémence est un comportement rationnel, ce qui suffit à définir une vertu : clementia rationi accidit ${ }^{67}$. Mais d'après Esprit, la raison livrée à elle-même souffre des faiblesses affectant l'entendement humain 68 . Le prince doit ainsi participer du projet céleste, et atteindre à une clémence qui ne se limite pas simplement à l'expression de la raison. Sénèque avait certes présenté la clementia comme une forme de l'imitatio deorum ${ }^{69}$, et comme une composante de l'amour du genre humain ${ }^{70}$. Mais Esprit va

65 H. Busson, La Religion des Classiques [n. 2], p. 210. Tallemant des Réaux, Historiettes, éd. A. ADAm, Paris, 1961, II, p. 351, estime déjà qu’il " n'est pas si dévot qu'on croit bien".

66 Cet aspect du De Clementia a été mis en lumière par J. BLÄNSDORF, “Seneca über Macht und Menschlichkeit", in Humanismus und Politik, hrsg. E. Olshausen, Stuttgart, 1983, p. 103-151 (e.g. p. 135-137). Esprit, I,10, p. 113-114, blâme Titus ou Nerva de n'être cléments que pour éviter les conjurations. Que la clementia protège des complots est justement l'un des leitmotive du De Clementia : I,8,6-7 ; I,9,12 ; I,25,3 ; I,26,1. De Clementia, II, 5,1 : "La clémence fait allégeance à la raison. "

68 La misère de la raison humaine devant le mystère divin est un thème éminemment janséniste, que saint Augustin objectait déjà aux Stoöciens (cf. e. g. JULIEN-EYMARD D’ANGERS “Réfutation... ” [n. 14], p. 377-378). Esprit lui-même dénonce l'orgueil de l'homme estimant sa Raison toute-puissante dès I,1. Sans Dieu, les projets de l'homme le plus habile sont voués à l'échec, notamment quand il entend instrumentaliser sa clémence à l'occasion, par exemple, d'une amnistie (I,1, p. 104).

M. Bellincioni, Potere ed etica in Seneca. Clementia e uoluntas amica, Brescia, 1984, p. 109. 
au-delà : la divinité n'est pas, comme chez Sénèque, un modèle d'ordre politique ${ }^{71}$, parmi bien d'autres; il n'est pas possible pour Esprit d'inviter "à régler les grands empires sur les petits 72 ». L'Éternel demeure l'unique source de perfection, si bien que la clementia principis doit, pour avoir quelque légitimité, être inspirée par l'amour du Seigneur. Les Anciens ignorent Dieu et en viennent à considérer le Sage comme l'égal des dieux ${ }^{73}$; partant, leur clémence est inacceptable. Cette dénonciation de la méconnaissance stoïcienne de la nature profonde des choses clôt le chapitre consacré à la clémence.

Concluons : la réflexion d'Esprit sur la clémence est manifestement nourrie de Sénèque. Esprit retourne contre ce dernier ses propres phrases, lui renvoie ses échecs, dénonce implicitement les illusions et les mensonges de sa thèse. Les deux conceptions comportent cependant bien des points communs, signes peut-être de la marque qu'a imprimée chez Esprit un commerce de toute une vie avec Sénèque. Cet apparent paradoxe n'est en rien une preuve de fatuité de la part d'Esprit : le rapport de la clémence à la divinité interdit à Esprit d'adhérer tout à fait à la doctrine du Stö̈cien. Celui-ci, conformément à une technique rhétorique éprouvée, présentait la clémence successivement du point de vue de l'honestum puis de l'utile, et faisait du gouvernement clément une entreprise conforme à la raison. Or, faire de la clementia un élément de l'honestum, ce n'est selon Esprit que flatter la vanité impériale, alors que l'utile dénonce l'insincérité d'une vertu reposant uniquement sur l'intérêt, la raison n'étant enfin que la marque de l'orgueil et de l'impuissance de l'homme.

Guillaume Flamerie de LACHAPELle

flamerie@club-internet.fr

UMR Ausonius

Université Michel-de-Montaigne/Bordeaux III

Domaine universitaire

33607 Pessac cédex

71 Cf. e. g. De Clementia, I,19,9. Sur ce point, cf. Tr. ADAM, Clementia principis [n. 41], p. 44 : "Die imitatio deorum wird nicht eigentlich... zu einer Frage der Religion, sondern der Moral und der Staatsraison. "

De Clementia, II,6,3. 